\title{
Considerations on coincidence experiments between two gravitational wave detectors for sources in the Galactic Centre
}

\author{
D. Babusci ${ }^{1}$, G. Giordano ${ }^{1}$, G. P. Murtas ${ }^{1}$, and G. Pizzella ${ }^{2,1}$ \\ 1 Laboratori Nazionali di Frascati dell'INFN, Frascati, Italy \\ e-mail: danilo.babusci@lnf.infn.it \\ 2 Università "Tor Vergata", Roma, Italy
}

Received 17 February 2004 / Accepted 8 May 2004

\begin{abstract}
In a coincidence experiment between gravitational wave detectors, the sidereal time analysis can provide very useful and powerful indications. We studied, with the help of a MonteCarlo simulation, the sidereal time pattern to be expected in such an experiment in the particular case of the resonant bars NAUTILUS and EXPLORER and for sources located in the Galactic Centre. It is shown that for linearly polarized gravitational waves the sidereal time pattern exhibits just a large peak at hour 3.5 and only a small peak at about hour 12 .
\end{abstract}

Key words. gravitational waves - methods: data analysis - methods: numerical

\section{Introduction}

Already Weber (Weber 1970) called the attention on the fact that gravitational wave $(\mathrm{GW})$ sources located in the Galactic Centre (GC) could generate in a resonant cylindrical detector signals at two sidereal hours separated exactly by twelve sidereal hours for detectors aligned along the east-west direction. In recent coincidence experiments between the two cryogenic resonant detectors EXPLORER and NAUTILUS (Astone et al. 2001,2002 ) an indication has been given, although not conclusive in terms of discovery of GWs, that a coincidence excess appeared around sidereal hour 4.3, as referred to the average of the detectors locations (EXPLORER: longitude $=6.20^{\circ} \mathrm{E}$, azimuth $=39^{\circ} ;$ NAUTILUS: longitude $=12.67^{\circ} \mathrm{E}$, azimuth $=$ $44^{\circ}$ ). In a sidereal time graph, just one peak showed up clearly, while one could have expected two identical peaks, which for EXPLORER and NAUTILUS should have occurred at 4.3 and 13.6 sidereal hours, under the hypothesis of GW sources concentrated at the GC. In an attempt to justify the occurrence of just one peak we considered the hypothesis that sources were distributed over the entire Galactic Disc.

In this note we wish to show that the experimental result of mainly one peak in a sidereal hour graph is consistent with the hypothesis that GW sources be concentrated in the GC and generate wave with a fixed polarization. We remind that the GW radiation emitted by any rotating quadrupole (for example a binary system) along the orbital plane, is linearly polarized in the same plane.

\section{Number of coincidences versus sidereal time}

As well known the cross-section of GW with a resonant detector depends on the direction of the wave and its polarization. Specifically, for a cylindrical detector with mass $M$ and material with sound velocity $v_{\mathrm{s}}$ we have (in units $\mathrm{m}^{2} \mathrm{~Hz}$ )

$\Sigma=\frac{8}{\pi} \frac{G M}{c}\left(\frac{v_{\mathrm{s}}}{c}\right)^{2} \sin ^{4} \theta \cos ^{2}(2 \chi)$

where $\theta$ is the angle between the detector axis and the GW direction, and $\chi$ is the angle between the detector axis and the polarization of the wave.

The cross-section allows to calculate the amount of energy absorbed by the detector

$E=\Sigma f(v)$,

where $f(v)$ is the $\mathrm{GW}$ spectral energy $\left(\mathrm{J} \mathrm{m}^{-2} \mathrm{~Hz}^{-1}\right)$ at frequency $v$.

We must realize that in a coincidence experiment only signals which exceed a given threshold are considered. For the Rome experiment the threshold is set at signal-to-noise ratio $S N R_{\mathrm{t}}=19.5$ (see Astone et al. 2001, 2002). If the absorbed energy generates a signal exceeding the threshold, then an event is produced, available for the coincidence search with events of another detector. Therefore, because of the dependence of the cross-section with the sidereal time, we expect, as a consequence of Eqs. (1) and (2), a sidereal time modulation of the number of events, both for a single detector and in a coincidence experiment.

In evaluating this modulation proper considerations need to be made. For example, if the GW signals are very large, so 


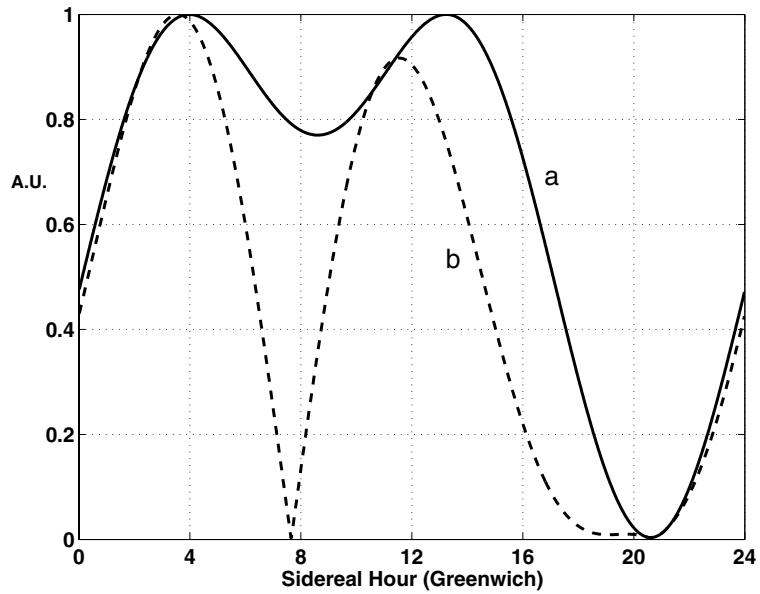

Fig. 1. NAUTILUS antenna pattern for sources in the GC: a) GW randomly polarized; b) GW with polarization parallel to the galactic plane.

that the absorbed energy exceeds the thresholds for most of the time, the modulation effect turns out to be barely apparent. Thus we can observe a clear modulation only if the signals have amplitude near the threshold, so that when the absorbed energy goes below the threshold then few or no events are generated. Calculations have already been made (Baryshev \& Paturel 2001; Paturel \& Baryshev 2003) for the expected sidereal time behavior of different detectors but not for coincidence experiments and without taking into account the effect of the noise. Indeed, one should also consider that the generation of an event depends strongly on the combination of the signal induced by the GW with the detector noise (Astone e al. 2000a), that gives the observed energy to be compared with the threshold. This is described by an efficiency parameter (see Astone et al. 2000b) $\epsilon(E)$, which is of the order of $50 \%$ for absorbed energy with signal-to-noise ratio $S N R_{\mathrm{s}}=S N R_{\mathrm{t}}$, and still of the order of $10 \%$ for $S N R_{\mathrm{S}} \sim S N R_{\mathrm{t}} / 2$. Thus, for a given detector, one has a number of events $N$ that is modulated with the sidereal time

$N(\theta, \chi) \propto \epsilon(E(\theta, \chi))$.

The average of this equation over $\chi$ (so over all possible polarizations) is called usually the antenna pattern of a detector with respect to a given source (see Fig. 1) ${ }^{1}$.

In a coincidence experiment between two detectors, the number of coincidences $N_{\mathrm{c}}$ goes with the product of the above $N_{\mathrm{s}}$ for the two detectors. Thus

$N_{\mathrm{c}}(\theta, \chi)=\epsilon_{1}(E(\theta, \chi)) \epsilon_{2}(E(\theta, \chi))$.

The coincidence pattern for randomly polarized sources, has to be obtained by averaging over $\chi$ Eq. (4), and not multiplying the two antenna patterns. The difference (see Fig. 2) comes from the fact that the responses of the two detectors are correlated, since the energy absorbed is fully determined by the

${ }^{1}$ In all the graphs we have used the sidereal time as referred to Greenwich longitude (GMST), since when considering the coincidences between different detectors, one has to use the same time scale for the events of all detectors. This choice of GMST seems the most natural one.

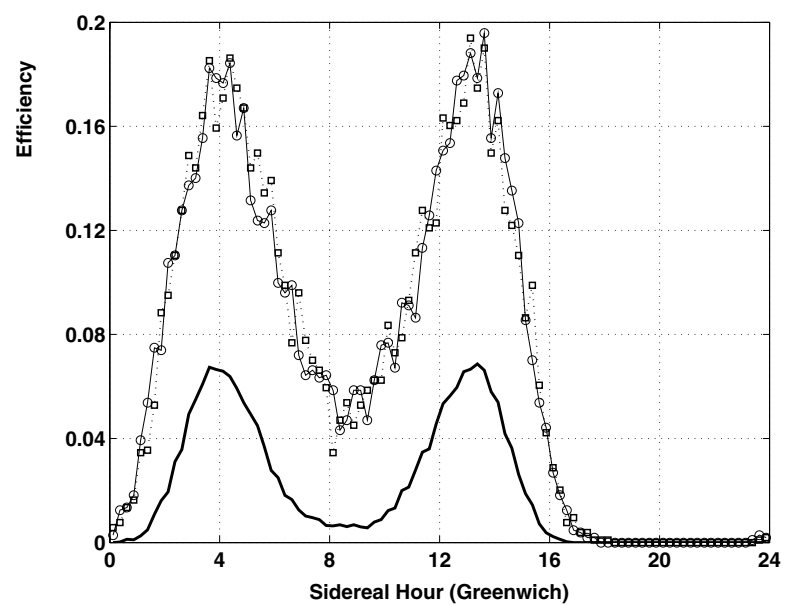

Fig. 2. Detection efficiency for NAUTILUS ( $\square$ ), EXPLORER (o) and their coincidences (thick solid line), for randomly polarized sources in the GC, with energy equal to the threshold. The average efficiencies are $\simeq 7 \%$ for a single detector and $\simeq 2 \%$ for the coincidence.

$\theta$ and $\chi$ angles, and only the combinations with the respective noises introduce an uncorrelation in the observed energies.

These considerations produce a time modulation of the number of coincidences which is in general very different from that obtained by simply plotting the behavior of the crosssection given by Eq. (1). If one does not properly take into consideration the above reasoning, he might obtain a false result.

\section{Polarized sources in the Galactic Centre}

As an example of how the source polarization can modify the coincidence pattern, we will consider here the case of a source located in the GC with polarization parallel to the galactic plane. Excluding the case of very large GW signals which would produce very small modulation if any, we consider two possible cases: a) GW signals with energy equal to the energy threshold for optimum direction and polarization $(\theta=\pi / 2$, $\chi=0$ ); b) GW signals with energy one half of the above case. In both cases, and also for the results shown in Fig. 2, the noise simulated in the MonteCarlo was such that the threshold, chosen with a $S N R=19.5$, was equal to (case a), or twice (case b), the value of the signal energy. We remark that both the ratio signal-energy/threshold and the noise value are important in determining the detection patterns.

From the graphs shown in Figs. 3 and 4 we note that the position of both peaks changes with respect to the randomly polarized case: the first peak moves slightly (from about hour 4 to 3.5 ), the second peak moves more (from about hour 13.5 to 11.5). Furthermore, both peaks are much narrower than those in Figs. 1 and 2. What is more important to note is that the single detector efficiencies already show a smaller number of events in the second peak with respect to the first one. This decreased efficiency, more pronounced for lower signal energies, becomes even more marked in the coincidence pattern, with a ratio of about 2.5 in the number of events present in the two peaks, thus making possible that in an experiment with a small number of total events detected, only the first peak could become visible. 

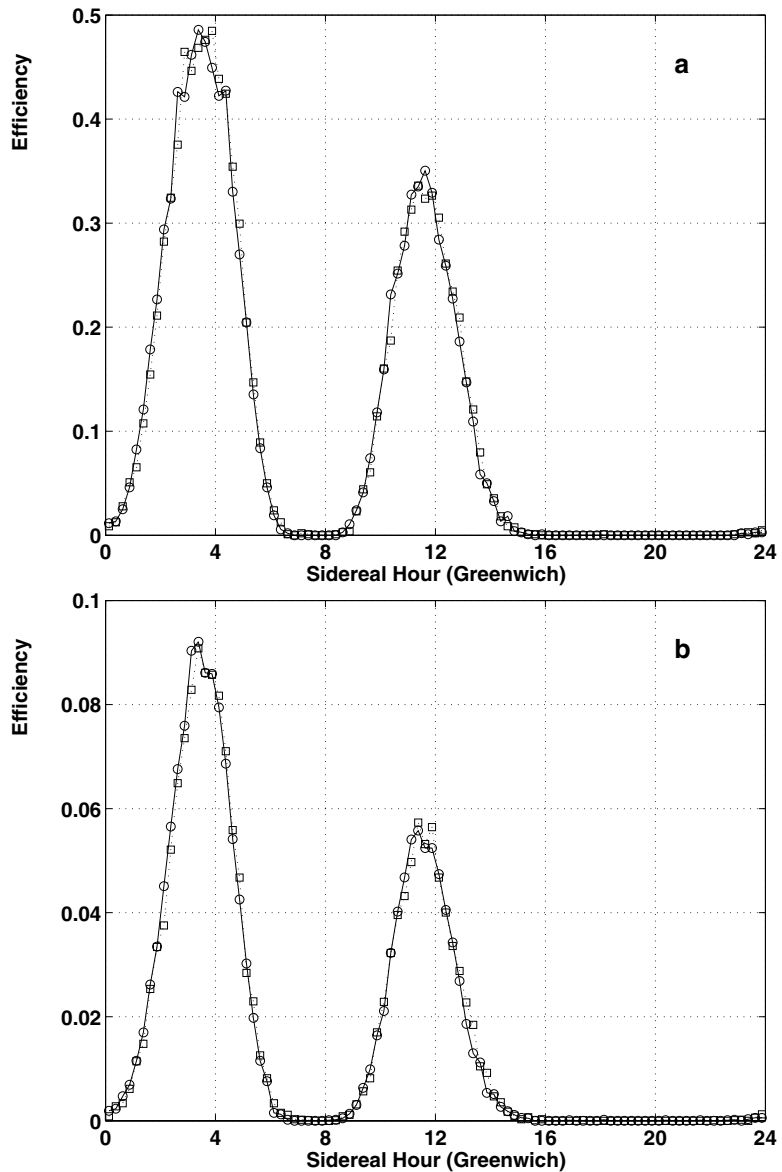

Fig. 3. Single detector (NAUTILUS ( $\square$ ) and EXPLORER (o)) efficiency for sources in the GC, polarization parallel to the galactic plane and signal energy equal to the threshold a) or half of the threshold $\mathbf{b}$ ). The average efficiency is $\simeq 10 \%$ a) and $\simeq 1.7 \%$ b).

\section{References}

Astone, P., Frasca, S., \& Pizzella, G. 2000a, Int. J. Mod. Phys. D, 9, 341

Astone, P., D’Antonio, S., \& Pizzella G. 2000b, Phys. Rev. D, 62, 042001

Astone, P., Bassan, M., Bonifazi, P., et al. 2001, Class. Quant. Grav., 18,243
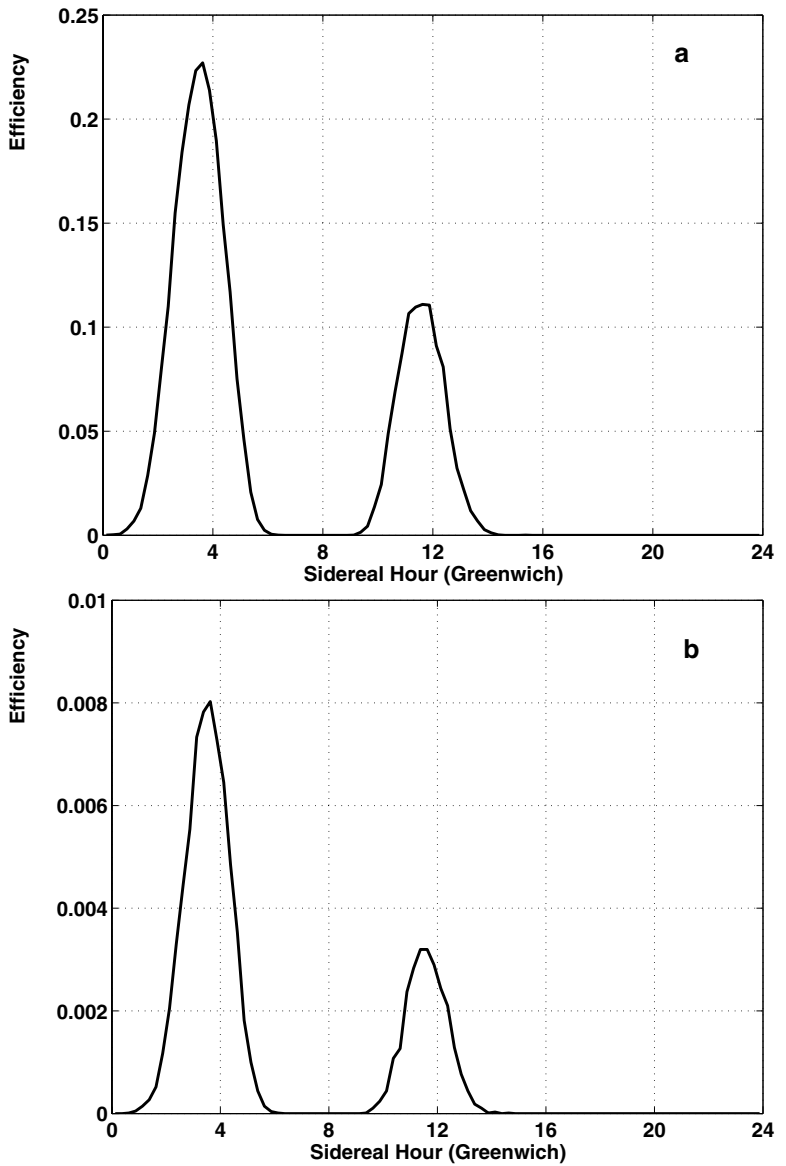

Fig. 4. Coincidence efficiency for the two cases of Fig. 3. The average efficiency is $\simeq 3.2 \%$ a) and $\simeq 0.1 \%$ b).

Astone, P., Babusci, D., Bassan, M., et al. 2002, Class. Quant. Grav., 19, 5449

Baryshev, Yu., \& Paturel, G. 2001, A\&A, 371, 378

Paturel, G., \& Baryshev, Yu. 2003, ApJ, 592, 199

Weber, J. 1970, Phys. Rev. Lett., 25, 180 